\title{
Inventory routing problems: an introduction
}

\author{
Luca Bertazzi • M. Grazia Speranza
}

Received: 11 June 2012/ Accepted: 2 November 2012

(C) Springer-Verlag Berlin Heidelberg and EURO - The Association of European Operational Research Societies 2012

\begin{abstract}
In this tutorial paper, we introduce the inventory routing problems (IRPs) with examples, we classify the characteristics of an IRP and present different models and policies for the class of problems where the crucial decision is when to serve customers. We call this class the problems with decisions over time only. The contributions are on the single link case, i.e., the problem where products are shipped from a supplier to a customer with capacitated vehicles, and on the IRPs with direct shipping. We overview the pioneering papers that appeared in the eighties, the literature on the single link and direct shipping problems, and cite the surveys and the tutorials available.
\end{abstract}

Keywords Tutorial $\cdot$ Survey $\cdot$ Single link $\cdot$ Direct shipping $\cdot$ Worst-case analysis

\section{Introduction}

It is well known that whenever a problem needs to be optimized, the decomposition of the problem into subproblems yields a sub-optimal solution. However, large problems are difficult to tackle and optimization models aim at optimizing a small part of a much more complex system. Whereas in several situations, the problem is relatively independent of the rest of the system, in other situations this is not the case. In general, modeling more comprehensive problems creates the opportunity for more savings.

In transportation and logistics, a trend in the direction of considering more and more comprehensive systems can be observed both in practice and in the academic world.

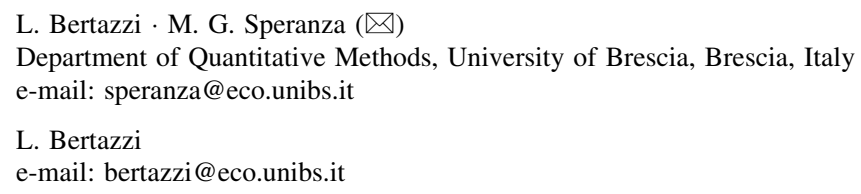


The concept of logistics has evolved over time to include more and more company functions and has inspired the concept of supply chain management that aims at including more companies in the integration process. The advance of information systems, the availability of data and the rise of the Internet have favored this direction.

Restricting the attention to optimization models in transportation and logistics, one can observe the growth of contributions in the modeling of systems that were traditionally decomposed and solved separately. This is also due to the advances in solution methods, both exact and heuristic, and the increased power of commercial software for linear programming (LP) and mixed integer linear programming (MILP) models.

In this paper, we introduce, motivate and survey the area of an inventory routing problems (IRPs), an area where the first contributions date back to the eighties and that has been constantly growing over time. The adoption by several companies of the so-called Vendor Managed Inventory (VMI) technique in supply chain management has partially driven the research in this area. Thousands of papers are available for the modeling and solution of routing problems and thousands of papers can be found on inventory management models. IRPs aim at integrating the two areas.

In this paper, we introduce the class of IRPs and focus on the decisions over time only, where the crucial decision is when to serve customers. In a forthcoming paper, we will focus on the decisions over time and space, where joint decisions on when to serve the customers and how to organize the routes are taken.

In "A motivating example" of this paper, we describe an example of a problem where a product has to be distributed to satisfy the demand of a network of customers over a discrete time period. The example is aimed at showing the impact on the transportation cost of taking joint decisions on whom to serve, how much to deliver and the routes to travel. In the example, the inventory cost plays no role. The main characteristics of an IRP, including the planning horizon, the policies, the objectives, the decisions, are presented and discussed in "Managing distribution networks". In "Decisions over time only: the Single Link Shipping Problem" and "Decisions over time only: the Inventory Routing Problem with Direct Shipping", we introduce and describe the main models for IRPs where the routing part has no role. In "Decisions over time only: the Single Link Shipping Problem", we define the single link problem and present different models, both in continuous and discrete time. The case of a distribution problem to several customers with direct shipping is treated in "Decisions over time only: the Inventory Routing Problem with Direct Shipping”. Finally, "Literature" is devoted to an overview of the literature. We cover the papers that appeared in the eighties and introduced in the literature the IRPs. We also cover the literature related to the specific content of this paper, i.e., the papers that tackle single link and direct shipping problems. Finally, we cite the surveys and the tutorials that cover the topic of this paper and of the follow-up paper.

\section{A motivating example}

In this section, we introduce the IRPs using an example inspired by one of the pioneering papers in the area, namely Bell et al. (1983). In this example, the 
inventory costs play no role. The example is aimed at showing the impact on the transportation cost of taking joint decisions on whom to serve, how much to deliver and the routes to travel.

The example

Time is assumed to be discrete, for example structured in days. A product has to be distributed from a supplier to a set of four customers with capacitated vehicles. Decisions have to be taken, for each day, on which customers to serve, how much to deliver and the routes to travel. There is no limitation on the availability of product at the supplier.

Let $M=\{1,2,3,4\}$ be the index set of the customers. Figure 1 shows the available connections between the supplier and the customers and between pairs of customers with their associated travel cost, i.e., the cost to pay if the connection is traveled by a vehicle. There is no limitation on the number of vehicles available. Each vehicle has capacity $C=5,000$. The daily demand $q_{s}$ of customer $s, s \in M$, is $1,000,3,000,2,000$ and 1,500. Each customer has a limited inventory level, i.e., the amount of product stocked at the customer cannot exceed this level. The maximum inventory level $U_{s}$ of customer $s, s \in M$, is 5,000, 3,000, 2,000 and 4,000. The initial inventory level of each customer $s$ is equal to its maximum inventory level, i.e., $I_{s 0}=U_{s}$, for all $s \in M$. No inventory cost is charged at the supplier and at the customers.

The objective is to find a periodic distribution policy, i.e., a plan on whom to serve, how much to deliver and the routes traveled by the vehicles, to be repeated regularly, that minimizes the total transportation cost. The policy must be such that a stock out is never caused at any of the customers, that the maximum inventory level at the customers is not exceeded and the vehicle capacity is satisfied. The periodicity of the policy implies that the inventory levels at the end of the period must be equal to the initial levels.

\section{A natural solution}

We first note that customers 2 and 3 must be served every day because their maximum inventory level equals the daily demand, i.e., they cannot stock more than the daily demand. Observing the traveling costs in Fig. 2, it is natural to combine the two pairs of close customers 1 and 2, and 3 and 4, and to serve daily each pair

Fig. 1 Bell et al. instance




with a separate vehicle. The vehicles capacity is not fully used. The daily cost is 420. Thus, the period of this policy is equal to 1 day.

\section{A better solution}

A better policy has period equal to 2 days. On the first day, customers 1 and 2 are served together with one vehicle and 3 and 4 are served together with another vehicle. A quantity 2,000 (twice the daily consumption) is delivered to $1,3,000$ (the daily consumption) to 2, 2,000 (the daily consumption) to 3 and 3,000 (twice the daily consumption) to 4 . The capacity of the vehicles is fully used in this case. Since customers 2 and 3 have to be served daily, due to their storage capacity and daily consumption, on the second day customers 2 and 3 are served together in a tour, 3,000 is delivered to 2 and 2,000 to 3 , while customers 1 and 4 are not visited. The average daily cost of this policy is 380 . It has been proved that this policy is optimal.

This example shows the impact of the decisions on whom to serve and how much to deliver on the transportation costs. The decisions on the routes of the vehicles have basically no impact on the costs as, given the customers to visit and the quantities to deliver, the routes are very easy to be found.

\section{Managing distribution networks}

We consider distribution networks with one supplier and one or several customers. In the inventory routing literature, the term 'retailer' is often used instead of the term 'customer'. This is due to the most common role of customers in VMI and to the fact that several papers in the inventory routing literature are motivated by the practical adoption of the VMI technique. In this paper, we will use the more general term 'customer'. We maintain the term 'supplier' to indicate the origin of the distribution flow. The supplier may identify a depot, a warehouse or a factory.

A set of products has to be shipped from the supplier to the customers to satisfy a deterministic demand over a time horizon that can be infinite or finite. Inventory
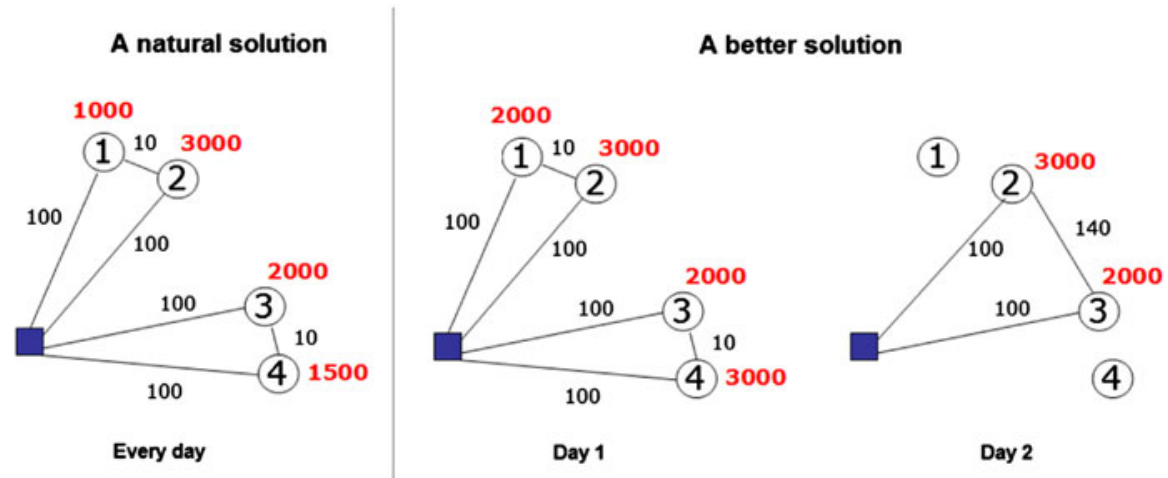

Fig. 2 Solutions for the Bell et al. instance 
costs are charged at the supplier and the customers. The situation of inventory costs at the supplier only or at the customers only are special cases. Shipments are performed by a fleet of vehicles having given capacity. A transportation cost is paid for each arc traversed by the vehicles.

In this section, we describe and structure the main characteristics of an IRP: the shipping times and the planning horizon, the structure of the distribution policy, the objective of the policy and the decision space. An optimization problem aims at determining an optimal policy where each of these characteristics is specified. Different specifications of these characteristics give rise to a different IRP.

Shipping times and planning horizon

The possible shipping times of an IRP can be:

1. Continuous A shipment can be performed at any time (starting from 0).

2. Continuous with a minimum intershipment time A shipment can be performed at any time (starting from 0), but the time between any pair of consecutive shipments (intershipment time) cannot be lower than a given minimum intershipment time, due to shipment/receiving set-up time requirements.

3. Discrete Shipments can be performed only at multiples of a minimum intershipment time. Since the minimum intershipment time can be normalized to 1 without loss of generality, shipments are performed at discrete times.

Let us denote by $z_{C}^{*}, z_{M}^{*}$ and $z_{D}^{*}$ the optimal cost of a problem with continuous shipping times, the optimal cost of a problem with continuous shipping times and minimum intershipment time and the optimal cost of a problem with discrete times, respectively. If all the other characteristics of the problem are the same, $z_{C}^{*} \leq z_{M}^{*} \leq z_{D}^{*}$. This ranking of the optimal values does not imply that the model with continuous shipping times should always be preferred to the others. In fact, for any specific situation, the most appropriate model should be chosen. Hall (1985), Maxwell and Muckstadt (1985), Jackson et al. (1988) and Muckstadt and Roundy (1993) pointed out the practical relevance of shipping policies where the intershipment times are discrete.

The planning horizon over which an optimal policy is looked at may be:

1. Infinite In this case, the IRP aims at determining a long-term distribution plan that may be useful, for example, to determine the fleet of vehicles, the number of drivers, and the organization of the distribution area in regions. More detailed operational problems may be solved at a later stage.

2. Finite The length of the planning horizon depends on the specific situation tackled. A short horizon is more operational than a long horizon.

\section{Structured policies}

Given the possible shipping times and the planning horizon, an optimal distribution policy is specified by the service times at the customers, the quantities to deliver and 
the routes of the vehicles. The problem of determining an optimal distribution policy, without any specific structure, may be extremely hard. Moreover, there may be practical reasons to restrict the structure of a policy. If the structure of a policy is defined, the aim of an IRP becomes finding an optimal or a heuristic policy in the class of policies having the given structure. Typical examples of structured policies, often inspired by practical relevance, are the following:

1. Zero Inventory Ordering (ZIO) Any customer is replenished if and only if its inventory level is down to zero.

2. Periodic A period $P$ has to be found. Any operation performed at time $t, 0 \leq t<P$, is repeated at times $t+k P, k=1,2, \ldots$.

3. Frequency-based These are periodic policies in which shipments are performed on the basis of one or several frequencies. For each frequency, the intershipment time is constant. In the single frequency policy, the intershipment time can be continuous or discrete. If more frequencies are allowed, then each frequency has an associated integer intershipment time and the period $P$ of the policy is the minimum common multiplier of the intershipment times.

4. Full load Shipments are performed using full load vehicles only.

5. Direct shipping Any customer is served independently by direct shipments from the supplier only. Routes that visit more than one customer are not allowed.

6. Order-up-to level Any customer has defined a maximum inventory level. Every time a customer is served, the delivered quantity is such that the maximum inventory level at the customer is reached.

7. Maximum level This class of policies generalizes the order-up-to level policies. Any customer has defined a maximum inventory level. Every time a customer is served, the delivered quantity is such that the inventory level at the customer is not greater than the maximum level.

8. Fixed partition The set of customers is partitioned into a number of sets such that each set is served separately and independently of the other sets. In other words, any route visits customers of the same set. The partition is typically based on the geographical location of the customers.

9. Partition-based This class of policies generalizes the fixed partition policies. Customers are partitioned into sets, as in the fixed partition policy. A route may visit customers of a set only, but also customers of specific combinations of two or more sets.

\section{Objectives}

The optimal solution of any IRP depends on the objective function chosen. The minimization of the transportation cost only is a suitable goal for a decision-maker who is responsible for the transportation only or for a situation where the inventory costs are not relevant when compared to the transportation costs. In this case, we may expect that an optimal solution prescribes infrequent transportation with highly loaded vehicles. The minimization of the inventory costs is the goal in situations where the focus is on inventory management. In this case, we may expect frequent transportation. This is what happens when just-in-time is implemented. The 
objective of minimizing the sum of the inventory and transportation costs is more suitable than the minimization of only one of the two cost components whenever a decision-maker is responsible for all the cost components. Tackling the transportation problem separately from the inventory management becomes in these cases a way to decompose a complex problem in simpler problems, but produces suboptimal solutions.

The following examples show that in the worst case, the solution obtained when the objective is to minimize the transportation cost only or the inventory cost only can be infinitely worse than the one obtained by minimizing the sum of the costs.

Example 1 Consider the case with one supplier and one customer. The shipping times are discrete. A set $I$ of products has to be shipped from the supplier to the customer. Each product $i \in I$ has a production and consumption rate $q_{i}$, a unit volume $v_{i}$ and unit inventory cost $h_{i}$. Let $\epsilon$ be such that $\frac{1}{\epsilon}$ is an integer number. Suppose that the total volume per time unit is $v=\sum_{i \in I} v_{i} q_{i}=\epsilon$ and the associated inventory cost is $h=\sum_{i \in I} h_{i} q_{i}=\frac{1}{\epsilon}$. The transportation is performed by vehicles having capacity $C=1$. The transportation cost per trip, i.e., the cost to go from the supplier to the customer and return to the supplier, is $c=100$.

Let us first compute the average total cost per time unit of a single frequency policy, i.e., a policy where a shipment takes place every $\tau$ times. The inventory cost at the supplier is computed as follows. Let us focus on any time interval of length $\tau$. As the production rate $q_{i}$ of any product $i \in I$ is constant over time, the total quantity produced every $\tau$ times is $q_{i} \tau$. Therefore, the total inventory level at the supplier over $\tau$ is $\frac{\left(q_{i} \tau\right) \tau}{2}$ and the average inventory level per time unit is $\frac{\left(q_{i} \tau\right) \tau}{2 \tau}=\frac{q_{i} \tau}{2}$. Since the unit inventory cost $h_{i}$ is charged for any product $i \in I$, the average inventory cost at the supplier is $\frac{\sum_{i \in I} h_{i} q_{i} \tau}{2}$ per time unit. Since the average inventory cost at the customer is identical, the total average inventory cost is $\sum_{i \in I} h_{i} q_{i} \tau$ per time unit. Let us now compute the transportation cost. As the total volume shipped every $\tau$ time is $v \tau, y=\left\lceil\frac{v \tau}{C}\right\rceil$, vehicles are needed every $\tau$ time. Therefore, the average transportation cost per time unit is $\frac{c}{\tau}\left\lceil\frac{v \tau}{C}\right\rceil$. Hence, the average total cost per time unit is $z^{S F}(\tau)=$ $\sum_{i \in I} h_{i} q_{i} \tau+\frac{c}{\tau}\left\lceil\frac{v \tau}{C}\right\rceil$.

Let us return to our numerical example. If the transportation cost only is taken into account, one fully loaded vehicle is sent every $\tau=\frac{1}{\epsilon}$ time with average total cost per time unit $z^{S F}\left(\frac{1}{\epsilon}\right)=\frac{1}{\epsilon^{2}}+100 \epsilon$. A different solution is obtained by sending one vehicle per time unit, with a resulting average total cost per time unit $z^{S F}(1)=$ $\frac{1}{\epsilon}+100$. Therefore,

$$
\frac{z^{S F}\left(\frac{1}{\epsilon}\right)}{z^{S F}(1)}=\frac{\frac{1}{\epsilon^{2}}+100 \epsilon}{\frac{1}{\epsilon}+100} \rightarrow \infty \quad \epsilon \rightarrow 0
$$

The above example shows that whenever the inventory costs are relevant but are ignored in the optimization, a very poor solution is likely to be found and implemented. 
Example 2 Consider again the problem of one supplier and one customer with discrete shipping times. Let $\epsilon$ be such that $\frac{1}{\epsilon}$ is an integer number. Let us assume that the total volume per time unit is $v=\epsilon$ and the total inventory cost per time unit is $h=\epsilon$. The transportation is performed by vehicles having capacity $C=1$ and cost per trip $c=\frac{1}{\epsilon}$.

If the objective chosen is the minimization of the inventory cost, one vehicle is sent per time unit. Therefore, the average total cost per time unit is $z^{S F}(1)=\epsilon+\frac{1}{\epsilon}$. A different solution can be obtained by sending one vehicle every $\frac{1}{\epsilon}$ time. The corresponding average total cost per time unit is $z^{S F}\left(\frac{1}{\epsilon}\right)=2$. Therefore,

$$
\frac{z^{S F}(1)}{z^{S F}\left(\frac{1}{\epsilon}\right)}=\frac{1+\frac{1}{\epsilon}}{2} \rightarrow \infty \quad \epsilon \rightarrow 0 .
$$

This example complements the previous one and shows that also ignoring the transportation costs may lead to very poor solutions.

\section{Decisions}

In vehicle routing problems, the customers to be visited are given as well as the quantities to be delivered. The decisions to be taken concern the routes of the vehicles, i.e., the space traversed by the vehicles. We call these decisions over space. If the customers of a supplier, independently of each other and independently of the supplier, decide when and how much to order, the problems to be solved by the supplier over time are vehicle routing problems. In this kind of traditional distribution management, that we call Retailer Managed Inventory (RMI), the power of the supplier to optimize the distribution is strongly constrained by the decisions taken by the customers, even when the goal is the minimization of the transportation cost only. Consider, for example, the case of two retailers located very close to each other, each ordering half a load once a week. The first retailer requests the delivery to take place on Monday and the second one the delivery to take place on Tuesday. The supplier needs to make two trips per week, with half load each. Clearly, if the supplier had the power to decide the timing of the deliveries, one full load trip only per week could be organized. The level of service to the retailers would remain the same and the cost would be halved. In VMI, the supplier has the power of deciding times and quantities of the deliveries. At the same time, it has the responsibility to avoid a stock-out at the retailers.

In inventory management, the decisions concern the timing and the quantities of the orders. We call these decisions over time.

In IRPs, the decision space always includes timing and quantities and may include the routing too. In general, we can classify the IRPs according to the decision space as follows:

1. Decisions over time only In this case, the routes are given. The decisions concern the times and the quantities to deliver to the customers. In the Single Link Shipping Problem, a supplier serves one customer only and, thus, the route traversed by the vehicles is given, from the supplier to the customer and back. 
Similarly, in the Inventory Routing Problem with Direct Shipping, a supplier serves a set of customers with direct shipments to each separately. Again, the routes are fixed.

2. Decisions over time and space The timing of the deliveries to each customer, the quantities to be delivered each time a delivery takes place and the routes traveled by the vehicles have to be decided at the same time.

This paper is restricted to the problems with decisions over time, whereas the problems with decisions over time and space will be the topic of a follow-up paper. The IRPs with decisions over time only have been deeply studied in the last two decades. The simplest problem of this type is the Single Link Shipping Problem. Its natural generalization to the case of several customers is the Inventory Routing Problem with Direct Shipping.

\section{Decisions over time only: the Single Link Shipping Problem}

A set $I$ of products has to be shipped from a supplier $A$ to a customer $B$. Each product $i \in I$ is made available at the supplier and absorbed at the customer at a constant rate $q_{i}$. Each unit of product $i \in I$ has a unit volume $v_{i}$ and a unit inventory $\operatorname{cost} h_{i}$ per time unit. The total volume produced per time unit is $v=\sum_{i \in I} v_{i} q_{i}$ and the total inventory cost per time unit is $h=\sum_{i \in I} h_{i} q_{i}$. The inventory level of any product $i \in I$ at the supplier and the customer must be always non-negative. Shipments are performed by a fleet of vehicles. Each vehicle has a transportation capacity $C$ (normalized to 1 , without loss of generality) and transportation cost $c$, charged for each trip independently of the quantity loaded on the vehicle. The goal of the problem is to find a shipping policy that minimizes the sum of the average transportation and inventory costs per time unit. The minimization of the transportation costs only is a special case obtained setting to 0 the unit inventory costs $h_{i}$.

In the following, we present different models for the Single Link Shipping Problem based upon different assumptions on the shipping times, the horizon and the structure of the policy.

As periodic policies play a relevant role, we define here the concept. A periodic policy is defined by the period $P$ and, in the continuous case, by the quantities $s_{i t}$ of product $i$ to be shipped at time $0 \leq t<P$, whereas, if time is discrete, by the quantities $s_{i t}$ to be shipped at times $t=0,1, \ldots, P-1$. Then, the number of vehicles $y_{t}$ to use at time $0 \leq t<P$ in the continuous case and at time $t=0,1, \ldots, P-1$ in the discrete case can be calculated. To define a periodic policy, the starting inventory levels $d_{i}^{A}$ and $d_{i}^{B}$ to make available at time 0 at the supplier and the customer are also needed. The quantity $s_{i t^{\prime}}=s_{i t}$ is then shipped at any time $t^{\prime}=$ $k P+t$, where $k$ is an integer $>0$.

An example of a periodic policy is the single frequency policy with discrete shipping times, where the products are shipped every $\tau$ times, with $\tau$ integer. In this case, $P=\tau, d_{i}^{A}=q_{i} \tau, d_{i}^{B}=0, s_{i 0}=q_{i} \tau, s_{i t}=0$ for $t=1,2, \ldots, P-1, y_{0}=\lceil\nu \tau\rceil$ and 
$y_{t}=0$ for $t=1,2, \ldots, P-1$. The corresponding average total cost per time unit is $h \tau+\frac{c}{\tau}\lceil v \tau\rceil$, which is identical to the cost $z(\tau)$ obtained in Example 1.

We will make use of the following remark, derived from the fact that demand and consumption are constant over time and equal at the supplier and the customer.

Remark 1 The sum of the inventory levels in $A$ and in $B$ is constant over time.

Thus, the total inventory level at any time $t$ is $d_{i}^{A}+d_{i}^{B}$.

The continuous case

In the continuous case, we assume that a shipment can be performed at any time and we aim at finding an optimal periodic policy. We can prove the following important property that characterizes the optimal policy.

Property 1 In the optimal periodic policy of the continuous case, the products are shipped at a single frequency, using a single vehicle.

Let us consider any periodic policy. Let $\bar{P}$ be the period of this policy, $\bar{\tau}$ be the maximum intershipment time and $\bar{m}$ be the number of vehicles used over a period. Let us show that the inventory cost is at least $h \bar{\tau}$. As there is no shipment in a time interval of length $\bar{\tau}$, the inventory level of each product $i \in I$ at the beginning of this time interval at the customer has to be at least $q_{i} \bar{\tau}$. Since the total (at the supplier and at the customer) inventory is constant over time, then the inventory level at any time is at least $\sum_{i} q_{i} \bar{\tau}$ and therefore the total inventory cost per time unit is at least $\sum_{i} h_{i} q_{i} \bar{\tau}=h \bar{\tau}$. Thus, the policy that minimizes the total inventory cost per time unit, given $\bar{P}$ and $\bar{m}$ (and therefore given the transportation cost), is the one with equally spaced intershipment times, i.e., the single frequency policy with intershipment time $\tau=\frac{\bar{P}}{\bar{m}}$.

Thanks to the characterization of the optimal periodic policy, the problem to find the optimal periodic policy can be formulated in the continuous case as the problem to determine the intershipment time $\tau_{C}^{*}$ such that the sum of the inventory and transportation costs is minimized. This problem, referred to as Problem $\mathcal{C}$, can be modeled as follows:

Problem $\mathcal{C}$

$$
\begin{gathered}
\min h \tau+\frac{c}{\tau} \\
\nu \tau \leq 1 \\
\tau \geq 0
\end{gathered}
$$

The objective function (1) expresses the minimization of the sum of the average transportation and the inventory costs per time unit. Constraint (2) is the capacity constraint and constraint (3) defines the non-negativity of the decision variable of the problem.

Problem $\mathcal{C}$ is a non-linear constrained optimization model and has the following simple closed solution (see Burns et al. 1985): 


$$
\tau_{C}^{*}=\min \left(\sqrt{\frac{c}{h}}, \frac{1}{v}\right) .
$$

When the optimal intershipment time is $\tau_{C}^{*}=\sqrt{\frac{c}{h}}<\frac{1}{v}$, then a vehicle is sent with partial load every $\tau_{C}^{*}$ time, whereas when $\tau_{C}^{*}=\frac{1}{v}$, a full load vehicle is sent. The model for the continuous case is very simple, but suffers from two main drawbacks. The first is that $\tau_{C}^{*}$ can be a very small number. The second is that $\tau_{C}^{*}$ can be an irrational number, such as $\sqrt{3}$. In both cases, the solution is impractical.

The continuous case with minimum intershipment time

To avoid that the intershipment time takes a very small impractical value, a minimum intershipment time, normalized to 1 , can be imposed. Such value takes into account shipment/receiving set-up time requirements. Since the structure of the optimal policy of this problem, called the continuous case with minimum intershipment time is unknown in this case, policies with a specific structure of practical relevance were proposed and analyzed. The simplest one is the single frequency policy. The optimal policy of this class can be obtained by solving the following model, where $y$ is the number of vehicles to use every $\tau$ times. This problem, referred to as Problem $\mathcal{M}^{S F}$, can be modeled as follows:

Problem $\mathcal{M}^{S F}$

$$
\begin{gathered}
\min h \tau+\frac{c}{\tau} y \\
\nu \tau \leq y \\
\tau \geq 1 \\
y \geq 0 \quad \text { integer. }
\end{gathered}
$$

The objective function (5) expresses the minimization of the sum of the average transportation and inventory costs per time unit. Constraint (6) is the capacity constraint. Constraint (7) takes into account the minimum intershipment time and (8) defines the decision variable $y$ of the problem.

Problem $\mathcal{M}^{S F}$ is a non-linear constrained optimization model and has the following optimal intershipment time (see Bertazzi et al. 2007)

$$
\tau_{M}^{*}=\min \left\{\max \left\{1, \sqrt{\frac{c}{h}\lceil\nu\rceil}\right\}, \frac{\lceil v\rceil}{v}\right\} .
$$

The corresponding optimal number of vehicles is $y^{*}=\left\lceil\nu \tau_{M}^{*}\right\rceil$.

A natural question is how much more expensive than the optimal policy the optimal single frequency policy is. This question may be partially answered by means of a worst-case analysis of the performance of the optimal single frequency policy with respect to the optimal policy. Let $z_{M}^{S F}$ be the optimal cost of Problem $\mathcal{M}^{S F}$, i.e., of the optimal single frequency policy, and $z_{M}^{*}$ be the cost of the optimal, unknown policy. The following result was proved in Bertazzi et al. (2007). 
Theorem $1 \frac{z_{M}^{S F}}{z_{M}^{*}} \leq \sqrt{2} \approx 1.414$ and the bound is tight.

This means that the optimal single frequency policy may increase the cost of the optimal one by up to $41.4 \%$. The fact that the bound is tight means that a smaller bound is not valid for all the problem instances because an instance exists where the bound is reached or a sequence of instances exists with ratio that tends to the bound. We now propose a different proof to give a complete example of worst-case analysis. Let us first prove that the worst-case ratio $\sqrt{2}$ holds for any instance of the problem.

Case $1 v \leq 1$ : in this case, if $c \geq h$, then $\tau_{M}^{*}=\tau_{C}^{*}$. Since $\tau_{C}^{*}$ is the optimal solution of a relaxed problem, shipping the products every $\tau_{M}^{*}$ is optimal. Otherwise, $\tau_{M}^{*}=1$ and $y^{*}=1$, with a corresponding cost $h+c$. This is optimal. In fact, any solution with at least one intershipment time $>1$ has inventory cost per time unit at least $2 h$. Since the minimum transportation cost per time unit is $c v$ (the cost in case of full load), the corresponding total cost per time unit is at least $2 h+c v>2 h>h+c$.

Case $2 v>1$ : in this case, $z_{M}^{S F}$ is not greater than the cost of shipping the products every $\tau_{c}=\sqrt{\frac{\lceil v\rceil}{v}}$ time using $\lceil v\rceil$ vehicles, i.e., $z_{M}^{S F} \leq h \sqrt{\frac{\lceil v\rceil}{v}}+\frac{c}{\sqrt{\frac{\lceil v\urcorner}{v}}}\lceil v\rceil=$ $\sqrt{\frac{\lceil v]}{v}}(h+c v)$.

Instead, $z_{M}^{*} \geq h+c v$, i.e., the sum of the minimum inventory cost (the cost to send the products every time unit) and the minimum transportation cost (the cost of a full load). Therefore,

$$
\frac{z_{M}^{S F}}{z_{M}^{*}} \leq \frac{\sqrt{\frac{\lceil v\rceil}{v}}(h+c v)}{h+c v} \leq \sqrt{\frac{\lceil v\rceil}{v}} \leq \sqrt{2},
$$

as $v>1$.

Since the worst-case ratio is obtained by overestimating $z_{M}^{S F}$ and underestimating $z_{M}^{*}$, one may think that the bound $\sqrt{2}$ could be reduced. This is not possible as there exists a sequence of instances such that the ratio between $z_{M}^{S F}$ and $z_{M}^{*}$ tends to $\sqrt{2}$. Let us consider the instance with volume per time unit $v=1+\epsilon$, where $\epsilon \leq \sqrt{2}-1$, and such that $\frac{1}{\sqrt{\epsilon}}$ is an integer number. The total average inventory cost per time unit is $h=1$. The transportation cost per trip $c$ is equal to 1 . The optimal solution of Problem $\mathcal{M}^{S F}$ is $\tau_{M}^{*}=\sqrt{2}$ and $y^{*}=2$, with a corresponding cost $z_{M}^{S F}=2 \sqrt{2}$. A different solution can be obtained by shipping a fraction $\frac{1}{1+\epsilon}$ every time unit and the remaining fraction $\frac{\epsilon}{1+\epsilon}$ every $\frac{1}{\sqrt{\epsilon}}$ time. Let $\tau_{1}=1$ and $\tau_{2}=\frac{1}{\sqrt{\epsilon}}$ be the corresponding intershipment times. The cost of this solution is $h\left(\frac{1}{1+\epsilon} \tau_{1}+\frac{\epsilon}{1+\epsilon} \tau_{2}\right)+\frac{c}{\tau_{1}}+\frac{c}{\tau_{2}}=\frac{1+\sqrt{\epsilon}}{1+\epsilon}+1+\sqrt{\epsilon}$. Therefore, $z_{M}^{*} \leq \frac{1+\sqrt{\epsilon}}{1+\epsilon}+1+\sqrt{\epsilon}$. Hence, in this sequence of instances, depending on $\epsilon$

$$
\frac{z_{M}^{S F}}{z_{M}^{*}} \geq \frac{2 \sqrt{2}}{\frac{1+\sqrt{\epsilon}}{1+\epsilon}+1+\sqrt{\epsilon}} \rightarrow \sqrt{2} \quad \epsilon \rightarrow 0 .
$$

Other specific policies were investigated in Bertazzi et al. (2007), namely the Best Single Frequency and the Best Double Frequency policies. 
In the Best Single Frequency, shipments are performed at the best possible frequency with discrete intershipment time. The Best Single Frequency policy is a particular case of the optimal single frequency. Whereas, the intershipment time of the optimal single frequency policy may be any continuous value $>1$, the intershipment time of the Best Single Frequency policy can only be a multiple of 1 . Therefore, the cost of the Best Single Frequency policy cannot be lower than the cost of the optimal single frequency policy. The reason to study the Best Single Frequency policy is that it is relevant from a practical point of view. In the Best Double Frequency policy, shipments are performed on the basis of the best two frequencies with discrete intershipment times. For example, one vehicle is sent every day and an additional vehicle every week.

Let $z^{S F}$ be the cost obtained by the Best Single Frequency policy. The following result holds:

Theorem $2 \frac{z^{S F}}{z_{M}^{*}} \leq \frac{5}{3} \approx 1.667$ and the bound is tight.

The proof of the worst-case ratio can be found in Bertazzi et al. (2007). The following example shows the instance that proves that the bound is tight.

Example 3 Consider the instance having volume $v=1+\epsilon$ per time unit, where $\epsilon$ is such that $\frac{1}{\sqrt{\epsilon}}$ is an integer number. The unit inventory cost per time unit is $h=1$. The transportation cost per trip $c$ is equal to 2 .

The cost of the single frequency policy with intershipment $\tau=1$ or $\tau=2$ is $h+2 c=2 h+\frac{3}{2} c=5$, while the cost with $\tau \geq 3$ is at least $3 h+c v>5$. Therefore, the Best Single Frequency policy has intershipment time either $\tau=1$ or $\tau=2$. A different solution can be obtained by shipping a fraction $\frac{1}{1+\epsilon}$ every time unit and the remaining fraction $\frac{\epsilon}{1+\epsilon}$ every $\frac{1}{\sqrt{\epsilon}}$ time. Let $\tau_{1}=1$ and $\tau_{2}=\frac{1}{\sqrt{\epsilon}}$ be the corresponding intershipment times. The cost of this solution is $h\left(\frac{1}{1+\epsilon} \tau_{1}+\frac{\epsilon}{1+\epsilon} \tau_{2}\right)+\frac{c}{\tau_{1}}+\frac{c}{\tau_{2}}=$ $\frac{1+\sqrt{\epsilon}}{1+\epsilon}+2+2 \sqrt{\epsilon}$. Therefore, in this instance

$$
\frac{z^{S F}}{z_{M}^{*}} \geq \frac{5}{\frac{1+\sqrt{\epsilon}}{1+\epsilon}+2+2 \sqrt{\epsilon}} \rightarrow \frac{5}{3} \quad \epsilon \rightarrow 0 .
$$

Let $z^{D F}$ be the cost of the Best Double Frequency policy. The following result holds:

Theorem $3 \frac{z^{D F}}{z_{M}^{*}} \leq \frac{16 \sqrt{3045}}{255}-\frac{37}{17} \approx 1.286$ and the bound is tight.

It can be shown that no reduction of the worst-case bound can be achieved by allowing more than two frequencies. The proof of these results can be found in Bertazzi et al. (2007), where practical policies have also been designed on the basis of these theoretical results. Computational results show that these policies are very effective. 
The discrete case

In the discrete case, the basic assumption is that shipments are performed at multiples of the minimum intershipment time, i.e., at discrete times if the minimum intershipment time is normalized to 1 .

A simple way to obtain a solution to this problem is to round-off the optimal solution $\tau_{C}^{*}$ of the continuous problem to an integer number. Three different heuristics based on this idea were proposed and analyzed in Bertazzi and Speranza (2001). In the first, referred to as Sup, $\tau_{C}^{*}$ is rounded-up; in the second, referred to as Inf, $\tau_{C}^{*}$ is rounded-down whenever $\tau_{C}^{*} \geq 1$, while it is rounded-up to 1 otherwise. Finally, in the third, referred to as Best, the solution at minimum cost between Sup and Inf is selected. Let $z^{\text {Round }}$ be the cost of any of these heuristics and $z_{D}^{*}$ be the optimal cost of the discrete case. The following result holds:

Theorem $4 \frac{z^{\text {Round }}}{z_{D}^{*}} \leq 2$ and the bound is tight.

This means that in the worst case, any of these rounding heuristics gives a percent increase in the total cost of $100 \%$ with respect to the optimal one. The proof of the worst-case performance bound can be found in Bertazzi and Speranza (2001). The following instance shows that the bound is tight.

Example 4 Consider the instance in which the volume per time unit is $v=1+\frac{1}{M}$, where $M \geq 2$ integer. The unit inventory cost per time unit is $h=\frac{1}{M^{2}}$. The transportation cost per trip $c$ is equal to 1 .

The optimal solution of Problem $\mathcal{C}$ is $\tau_{C}^{*}=\frac{M}{M+1}$. Since $\tau_{C}^{*}<1$, the heuristics Sup, Inf and Best send the products every time unit using two vehicles, with a corresponding cost $h+2 c=\frac{1}{M^{2}}+2$. A different solution can be obtained by sending the products every $M$ time using $M+1$ vehicles. The cost of this solution is $h M+\frac{c}{M}(M+1)=\frac{2}{M}+1$. Therefore, for this instance

$$
\frac{z^{\text {Round }}}{z_{D}^{*}} \geq \frac{\frac{1}{M^{2}}+2}{\frac{2}{M}+1} \rightarrow 2 \quad M \rightarrow \infty .
$$

A better way to solve this problem is to apply the Best Single Frequency policy. The worst-case performance bound of this policy is simple to be obtained. Indeed, $z_{M}^{*} \leq z_{D}^{*} \frac{z^{S F}}{z_{D}^{*}} \leq \frac{z^{S F}}{z_{M}^{*}} \leq \frac{5}{3} \approx 1.667$. Moreover, as in the instance shown in Example 3, only solutions with discrete shipping times are considered, the bound is tight.

A practical policy, called Full Load, whose behavior is worth investigating, is to use fully loaded vehicles at discrete times. A shipment is performed at the first discrete time not lower than when a full load is reached. Bertazzi and Speranza (2005) showed that this policy generates in the worst case an infinitely large error with respect to the optimum, i.e., there exists at least one instance such that $\frac{z^{\text {Full }}}{z_{D}^{*}} \rightarrow$ $\infty$, where $z^{\text {Full }}$ is the cost of the Full Load policy. However, there exist classes of instances where $\frac{z^{\text {Full }}}{z_{D}^{*}} \leq 2$ and $\frac{z^{\text {Full }}}{z_{D}^{*}} \leq \frac{3}{2}$. In particular, the $\frac{3}{2}$ bound holds for all the 
instances where the full load policy is optimal in the continuous problem, i.e., when $\tau_{C}^{*}=\min \left(\sqrt{\frac{c}{h}}, \frac{1}{v}\right)=\frac{1}{v}$. For this class of instances, the Full Load policy is better than the Best Single Frequency policy.

The best theoretical performance of a policy was obtained in Bertazzi and Chan (2011) for the case with a single product, by applying the Best Double Frequency policy. The authors showed that $\frac{z^{D F}}{z_{D}^{*}} \leq \frac{123}{106} \approx 1.1603$, that the bound is tight and no improvement can be obtained by allowing more than two frequencies.

The discrete case with given frequencies

The concept of frequency is commonly used in transportation. A service may be provided daily, or weekly, or monthly, sometimes more often, for example twice a day. The use of frequency is well accepted in practice because of the regularity of a frequency-based process that simplifies the organization of both the supplier and the customer. We have already used the concept of frequency for the Single and Double Frequency policies. We present here an optimization model that, given a set of possible frequencies, identifies the best ones. This problem is of interest when the possible shipment frequencies are not defined by the decision-maker, but by third parties.

The problem was introduced in Speranza and Ukovich (1994) and studied in Speranza and Ukovich (1996), Bertazzi et al. (2000) and Bertazzi and Speranza (2002). Each frequency $f_{j}, j \in J$, is such that the corresponding intershipment time $t_{j}=1 / f_{j}$ is discrete. Therefore, shipments are performed at discrete times. The period of this policy is $P=L C M\left\{t_{j}, j \in J\right\}$, where LCM is the Least Common Multiplier. Let $x_{i j}$ be the percentage of product $i \in I$ to ship at frequency $j \in J$ and $y_{j}$ be the number of vehicles to use at frequency $j \in J$. The optimization model for this problem, called Problem $\mathcal{F}$, is

Problem $\mathcal{F}$

$$
\begin{gathered}
\min \sum_{i \in I} \sum_{j \in J} h_{i} q_{i} t_{j} x_{i j}+\sum_{j \in J} \frac{c}{t_{j}} y_{j} \\
\sum_{j \in J} x_{i j}=1 \quad i \in I \\
\sum_{i \in I} v_{i} q_{i} t_{j} x_{i j} \leq y_{j} \quad j \in J \\
x_{i j} \geq 0 \quad i \in I \quad j \in J \\
y_{j} \geq 0 \quad \text { integer } \quad j \in J
\end{gathered}
$$

The objective function (10) expresses the minimization of the sum of the average transportation and inventory costs per time unit. Constraints (11) guarantee that the products are totally shipped from the supplier to the customer using one or several frequencies. Constraints (12) guarantee that the number of vehicles used at each frequency is sufficient. Finally, constraints (13) and (14) define the decision variables. 
Exact and heuristic algorithms were proposed in Speranza and Ukovich (1996) and Bertazzi et al. (2000) for the solution of this problem. In the latter paper, a large set of instances with up to 10,000 different products are solved to optimality. Moreover, while it is computationally shown that simple heuristics may fail to find good quality solutions, an effective heuristic is proposed that produces solutions with average error $<0.3 \%$ with respect to the optimal solution.

The discrete case with finite time horizon

A supplier may be sufficiently flexible to accept that any discrete time is a possible shipping time and be interested in finding the best shipping times over a finite planning period.

The optimization model for this problem was introduced in Bertazzi and Speranza (2002). A finite time horizon $H$ is given. The set of possible shipping times is $T=\{0,1, \ldots, H-1\}$. The problem is to determine the quantity $s_{i t}$ of each product $i \in I$ to ship at each time $t \in T$, the starting inventory level $d_{i}^{A}$ and $d_{i}^{B}$ to make available at the supplier $A$ and the customer $B$ and the number of vehicles $y_{t}$ to use at each time $t \in T$, in order to minimize the sum of the transportation and inventory costs over the time horizon $H$. The optimization problem, referred to as Problem $\mathcal{H}$, can be formulated as follows:

Problem $\mathcal{H}$

$$
\begin{gathered}
\min \sum_{i \in I} h_{i}\left(d_{i}^{A}+d_{i}^{B}\right)+\sum_{t \in T} \frac{c}{H} y_{t} \\
\sum_{t \in T} s_{i t}=q_{i} H \quad i \in I \\
\sum_{i \in I} v_{i} s_{i t} \leq y_{t} \quad t \in T \\
d_{i}^{A}+q_{i} t-\sum_{k=0}^{t} s_{i k} \geq 0 \quad i \in I \quad t \in T \\
d_{i}^{B}+\sum_{k=0}^{t} s_{i k}-q_{i}(t+1) \geq 0 \quad i \in I \quad t \in T \\
d_{i}^{A} \geq 0 \quad d_{i}^{B} \geq 0 \quad i \in I \\
s_{i t} \geq 0 \quad i \in I \quad t \in T \\
y_{t} \geq 0 \quad \text { integer } \quad t \in T
\end{gathered}
$$

The objective function (15) expresses the minimization of the sum of the inventory and transportation costs. Constraints (16) guarantee that each product is totally shipped from the supplier to the customer. Constraints (17) guarantee that the number of vehicles used at each time $t$ is sufficient. Inequalities (18) and (19) are the stock-out constraints in $A$ and in $B$. Finally, (20)-(22) define the decision variables. 
Let $z_{H}^{*}$ be the optimal cost of Problem $\mathcal{H}$. If the period of Problem $\mathcal{F}$ and Problem $\mathcal{H}$ is $H$, then Problem $\mathcal{F}$ is a particular case of Problem $\mathcal{H}$. Therefore, $z_{H}^{*} \leq z_{F}^{*}$. Moreover, let $z_{F}^{*}(H)$ be the optimal cost of Problem $\mathcal{F}$ when the set of frequencies is composed of all frequencies which are submultiples of $H$. In this case, there exists an instance such that $\frac{z_{F}^{*}(H)}{z_{H}^{*}} \rightarrow \infty$ (see Bertazzi and Speranza 2002).

\section{Decisions over time only: the Inventory Routing Problem with Direct Shipping}

When a supplier has several customers, the problem of serving them becomes more complex. It is interesting to investigate the performance of direct shipments, i.e., shipments that visit one customer only at a time. This policy may be very practical and effective when the demand of the customers is large. We call this problem the Inventory Routing Problem with Direct Shipping.

The effectiveness of direct shipments was studied by Gallego and Simchi-Levi (1990) in the case of continuous shipping times. It was shown that the worst-case performance ratio of direct shipping with respect to a lower bound on the optimal cost is no more than about 1.061 whenever the Economic Lot Size of each customer is at least $71 \%$ of the vehicle capacity. Comments and extensions of this analysis can be found in Hall (1992), Gallego and Simchi-Levi (1994) and Jones and Qian (1997).

Bertazzi (2008) studied the performance of direct shipping policies where each link is optimized independently, for the case with discrete shipping times. In the worst case, the ratio between the cost of the best direct shipping policy and the optimal cost of the problem where routing is allowed is not greater than 2 whenever the volume per time unit on each link is not lower than $1 / 4$ of the vehicle capacity. Moreover, if the volume is not lower than the capacity, the bound is approximately 1.21. These bounds are tight, obtained by making use of at most three shipping frequencies and cannot be improved using different policies.

\section{Literature}

In this section, we briefly overview a set of pioneering papers, i.e., the set of papers that appeared in the eighties, and introduced in the literature the first IRPs starting from real applications. We also overview the literature discussed in this paper, i.e., the papers that tackle direct shipping problems. Finally, we cite the surveys and the tutorials on IRPs that cover all the material of this paper and of the forthcoming paper focused on the decisions over time and space.

\section{Pioneering papers}

The expression inventory routing appeared in the literature in a series of papers published in the eighties (Bell et al. 1983; Federgruen and Zipkin 1984; Golden et al. 1984; Blumenfeld et al. 1985; Burns et al. 1985; Hall 1985; Maxwell and 
Muckstadt 1985; Roundy 1985; Dror et al. 1985; Dror and Ball 1987). In these papers, a variety of different problems that were motivated by real applications were presented. The common element to the problems was the focus on the joint optimization of transportation and inventory management.

Single link problem

The single link problem has been discussed in depth in this paper. We summarize here the papers where the problem was studied. The first model for the single link case, namely the discrete case with given frequencies, was presented in Speranza and Ukovich (1994) and was motivated by a real application. A subsequent series of papers followed that paper: Speranza and Ukovich (1996), Bertazzi et al. (2000, 2007), Bertazzi and Speranza (2001, 2002, 2005a, b), Solyalı and Süral (2008), Bertazzi and Chan (2011).

Only one paper, namely Bertazzi et al. (2001), is available where the stochasticity of the demand in a single link problem is modeled.

\section{Direct shipping}

The problem of evaluating the performance of direct shipping in a distribution problem from supplier to several customers was studied in Gallego and Simchi-Levi (1990), Gallego and Simchi-Levi (1994), Hall (1992), Jones and Qian (1997), and Bertazzi (2008).

Surveys and tutorials

Several surveys on IRPs were published, confirming the level of attention that the area has attracted in the last decades. The first survey was published by Federgruen and Simchi-Levi (1995), and was followed by several others: Campbell et al. (1998), Baita et al. (1998), Campbell et al. (2002), Moin and Salhi (2007). Finally, two tutorials were published in the last years: Bertazzi et al. (2008) and Bertazzi and Speranza (2011).

Acknowledgments We are grateful to two anonymous reviewers who have helped us improve an earlier version of this paper.

\section{References}

Baita F, Ukovich W, Pesenti R, Favaretto D (1998) Dynamic routing-and-inventory problems: a review. Transp Res Part A Policy Pract 32(8):585-598

Bell WJ, Dalberto LM, Fisher ML, Greenfield AJ, Jaikumar R, Kedia P, Mack RG, Prutzman PJ (1983) Improving the distribution of industrial gases with an on-line computerized routing and scheduling optimizer. Interfaces 13:4-23

Bertazzi L (2008) Analysis of direct shipping policies in an inventory-routing problem with discrete shipping times. Manag Sci 54(4):748-762

Bertazzi L, Chan LMA (2011) Analysis of the best double frequency policy in the single link problem with discrete shipping times. Technical Report n. 366, Dept. of Quantitative Methods, University of Brescia (submitted) 
Bertazzi L, Speranza MG (2001) Rounding procedures for the discrete version of the capacitated economic order quantity problem. Ann Oper Res 107(1):33-49

Bertazzi L, Speranza MG (2002) Continuous and discrete shipping strategies for the single link problem. Transp Sci 36(3):314-325

Bertazzi L,Speranza MG (2005a) Improved rounding procedures for the discrete version of the capacitated EOQ problem. Eur J Oper Res 166(1):25-34

Bertazzi L, Speranza MG (2005b) Worst-case analysis of the full load policy in the single link problem. Int J Prod Econ 93:217-224

Bertazzi L, Speranza MG (2011) Matheuristics for inventory routing problems. In: Montoya-Torres JR, Juan AA, Huatuco LH, Faulin J, Rodriguez-Verjan GL (eds) Hybrid algorithms for service, computing and manufacturing systems: routing and scheduling solutions. IGI Global, Hershey, pp $1-14$

Bertazzi L, Speranza MG, Ukovich W (2000) Exact and heuristic solutions for a shipment problem with given frequencies. Manag Sci 46:973-988

Bertazzi L, Bertsekas DP, Speranza MG (2001) Optimal and neuro-dynamic programming solutions for a stochastic inventory transportation problem. In: Kischka P, Wildburger UL, Radermacher FJ (eds) Models, methods and decision support for management. Springer, Berlin, pp 65-78

Bertazzi L, Chan LMA, Speranza MG (2007) Analysis of practical policies for a single link distribution system. Naval Res Logist 54(5):497-509

Bertazzi L, Savelsbergh M, Speranza MG (2008) Inventory routing. In: Golden B, Raghavan S, Wasil $\mathrm{E}$ (eds) The vehicle routing problem: latest advances and new challenges. Springer, Berlin, pp 49-72

Blumenfeld DE, Burns LD, Diltz JD, Daganzo CF (1985) Analyzing trade-offs between transportation, inventory and production costs on freight networks. Transp Res Part B Methodol 19(5):361-380

Burns LD, Hall RW, Blumenfeld DE, Daganzo CF (1985) Distribution strategies that minimize transportation and inventory costs. Oper Res 33:469-490

Campbell A, Clarkec L, Kleywegt A, Savelsbergh M (1998) The inventory routing problem. In: Crainic TG, Laporte G (eds) Fleet management and logistics. Kluwer, USA, pp 95-113

Campbell A, Clarke L, Savelsbergh MWP (2002) Inventory routing in practice. The vehicle routing problem. In: Toth P, Vigo D (eds) SIAM monographs on discrete mathematics and applications, vol 9, pp 309-330

Dror M, Ball M (1987) Inventory/routing: reduction from an annual to a short-period problem. Naval Res Logist 34(6):891-905

Dror M, Ball M, Golden B (1985) A computational comparison of algorithms for the inventory routing problem. Ann Oper Res 4(1):1-23

Federgruen A, Simchi-Levi D (1995) Analysis of vehicle routing and inventory-routing problems. In: Ball MO, Magnanti TL, Monma CL, Nemhauser GL (eds) Handbooks in operations research and management science, vol 8. Elsevier, Amsterdam, pp 297-373

Federgruen A, Zipkin P (1984) A combined vehicle routing and inventory allocation problem. Oper Res 32:1019-1037

Gallego G, Simchi-Levi D (1990) On the effectiveness of direct shipping strategy for the one-warehouse multi-retailer R-systems. Manag Sci 36:240-243

Gallego G, Simchi-Levi D (1994) Rejoinder to 'A note on bounds for direct shipping costs'. Manag Sci 40(10):1393

Golden B, Assad A, Dahl R (1984) Analysis of a large scale vehicle routing problem with an inventory component. Large Scale Systems 7(2-3):181-190

Hall RW (1985) Determining vehicle dispatch frequency when shipping frequency differs among suppliers. Transp Res Part B Methodol 19(5):421-431

Hall RW (1992) A note on bounds for direct shipping cost. Manag Sci 38(8):1212-1214

Jackson PL, Maxwell WL, Muckstadt JA (1998) Determining optimal reorder intervals in capacitated production-distribution systems. Manag Sci 34:938-958

Jones PC, Qian T (1997) Fully loaded direct shipping strategy in one warehouse/N retailer systems without central inventories. Transp Sci 31(2):193-195

Maxwell WL, Muckstadt JA (1985) Establishing consistent and realistic reorder intervals in productiondistribution systems. Oper Res 33:1316-1341

Moin NH, Salhi S (2007) Inventory routing problems: a logistical overview. J Oper Res Soc 58(9):1185-1194 
Muckstadt JA, Roundy RO (1993) Analysis of multistage production systems. In: Graves SC, Rinnooy Kan AHG, Zipkin PH (eds) Handbooks in operations research and management science, vol 4. Elsevier, Amsterdam, pp 59-131

Roundy R (1985) 98 \%-effective integer-ratio lot-sizing for one-warehouse multi-retailer systems. Manag Sci 31:1416-1430

Solyalı O, Süral H (2008) A single supplier-single retailer system with an order-up-to level inventory policy. Oper Res Lett 36(5):543-546

Speranza MG, Ukovich W (1994) Minimizing transportation and inventory costs for several products on a single link. Oper Res 42:879-894

Speranza MG, Ukovich W (1996) An algorithm for optimal shipments with given frequencies. Naval Res Logist 43(5):655-671 